\title{
Seasonal Selection Preferences for Woody Plants by Breeding Herds of African Elephants (Loxodonta africana) in a Woodland Savanna
}

\author{
J. J. Viljoen, ${ }^{1}$ H. C. Reynecke, ${ }^{1}$ M. D. Panagos, ${ }^{1}$ W. R. Langbauer Jr., ${ }^{2}$ and A. Ganswindt ${ }^{3,4}$ \\ ${ }^{1}$ Department of Nature Conservation, Tshwane University of Technology, Private Bag X680, Pretoria 0001, South Africa \\ ${ }^{2}$ Buttonwood Park Zoo, New Bedford, MA 02740, USA \\ ${ }^{3}$ Department of Zoology and Entomology, University of Pretoria, Pretoria 0002, South Africa \\ ${ }^{4}$ Department of Production Animal Studies, Faculty of Veterinary Science, University of Pretoria, Onderstepoort 0110, South Africa
}

Correspondence should be addressed to J. J. Viljoen; viljoenjj@tut.ac.za

Received 19 November 2012; Revised 25 February 2013; Accepted 25 February 2013

Academic Editor: Bruce Leopold

Copyright (C) 2013 J. J. Viljoen et al. This is an open access article distributed under the Creative Commons Attribution License, which permits unrestricted use, distribution, and reproduction in any medium, provided the original work is properly cited.

To evaluate dynamics of elephant herbivory, we assessed seasonal preferences for woody plants by African elephant breeding herds in the southeastern part of Kruger National Park (KNP) between 2002 and 2005. Breeding herds had access to a variety of woody plants, and, of the 98 woody plant species that were recorded in the elephant's feeding areas, 63 species were utilized by observed animals. Data were recorded at 948 circular feeding sites (radius $5 \mathrm{~m}$ ) during wet and dry seasons. Seasonal preference was measured by comparing selection of woody species in proportion to their estimated availability and then ranked according to the Manly alpha $(\alpha)$ index of preference. Animals demonstrated a selection preference in feeding on woody vegetation, and Grewia hexamita, Grewia bicolor, Grewia flavescens, and Grewia monticola were selected consistently more over all seasons. In addition, our results indicate that elephant herds have a low preference for at least some of the woody species prone to extirpation and that feeding preferences for woody plants do not account for the association of elephants and riparian fringe habitat.

\section{Introduction}

The transformed and fragmented South African landscape has resulted in a lack of suitably sized protected areas for elephants, thus creating major challenges for managing elephant populations relative to their effects on ecosystem processes, vegetation structure, and species of plants and other animals [1]. Prolonged periods of locally high densities of elephants can alter vegetation structure, and the impact can intensify, especially when animals are confined to small or medium sized fenced reserves $[2,3]$. This is especially challenging in fenced reserves smaller than $1000 \mathrm{~km}^{2}$ to which elephants have been reintroduced by 2001 in South Africa [4, 5]. Subsequent to the introduction of elephant in these small areas, these savannas are exposed to intensive management, and decision makers are increasingly challenged to choose options which can balance needs of locally high elephant numbers with the maintenance of vegetation and ecosystem diversity.

Elephant feeding behaviour in combination with natural processes, for example, climate and fire, is the architects in different African landscapes and has been the focus of numerous studies describing elephant diet [6-11]. However, many of these studies failed to define selection preferences at the plant species level and only describe preferences at the broad level of growth forms $[12,13]$. This gap resulted in incorrectly assigning certain responses in the environment to elephants [14]. White and Goodman [15] used quantitatively collected field data and concluded that differences in the structure and the diversity of woody vegetation in Mkhuze Game Reserve, South Africa, were unrelated to elephant use. Similarly, Landman et al. [16] used a microhistological analysis of faecal material to determine composition of elephant diet and thereby refuted the popular belief that elephant 
herbivory was the major driver of decline of important plants in the Addo Elephant National Park, South Africa.

Food resources are distributed heterogeneously across the landscape and vary in quality and quantity between seasons [14] which might explain elephants altering their selection from green grass during wet seasons to browse foliage during dry seasons [18-20]. Therefore, it could be assumed that elephants might feed through a wide variety of vegetation communities for food species that permit efficient and effective nutrient intake and that specific plants meeting these criteria vary seasonally. Currently, only limited information is available regarding seasonal-dependent variability in food of African elephants, although such information would be important to avoid making suboptimal management decisions.

We describe the seasonal relative availability and speciesspecific preference for woody vegetation by free-ranging African elephant breeding herds in the southern part of the Kruger National Park (KNP).

\section{Material and Methods}

2.1. Study Area. Data were collected from March 2002 to September 2005 in the KNP within the Granite Lowveld, Delagoa Lowveld, Tshokwane-Hlane Basalt Lowveld, Gabbro Grassy Bushveld, and the Northern Lebombo Bushveld [21]. The KNP covers an area of $19000 \mathrm{~km}^{2}$ and can be longitudinally divided into resistant granites in the west succeeded by Ecca shales, basalt, and rhyolites in the east that give rise to different soil types and associated flora and fauna [22]. Average rainfall varies from 500 to $750 \mathrm{~mm} /$ year [23]. From May to July is the early dry season, the late dry season is from August to October, the early wet season is from November to January, and the late wet season is from February to April [24].

2.2. Study Animals. We selected, as our focal herd, the first breeding herd of elephants in the southern region of KNP spotted from a helicopter after the take-off. On January 14, 2001, the matriarch was fitted with a VHF radio collar (Africa Wildlife Tracking cc, South Africa) which was replaced in August 2002 with a GPS satellite collar (Africa Wildlife Tracking cc, South Africa) for easier location. We trailed the breeding herds on foot which provided us the means to study patterns of woody plant use in all terrains, thus eliminating the sample bias that would be introduced if elephants were studied only in areas where elephants were regularly seen or areas that were easily accessible by road. To ensure that the collared group's feeding behaviour was not atypical, following the same protocol as above, we recorded selection of noncollared breeding herds randomly sighted in the study area. To avoid collecting from the same breeding herds, we included data from about 20 different herds. Different individuals joined and left the core group from time to time, and contacts with these breeding herds were not continuous throughout the study period. Family groups (breeding herds) are defined as individuals who are seen together more than $70 \%$ of the time [25]. We did not collect

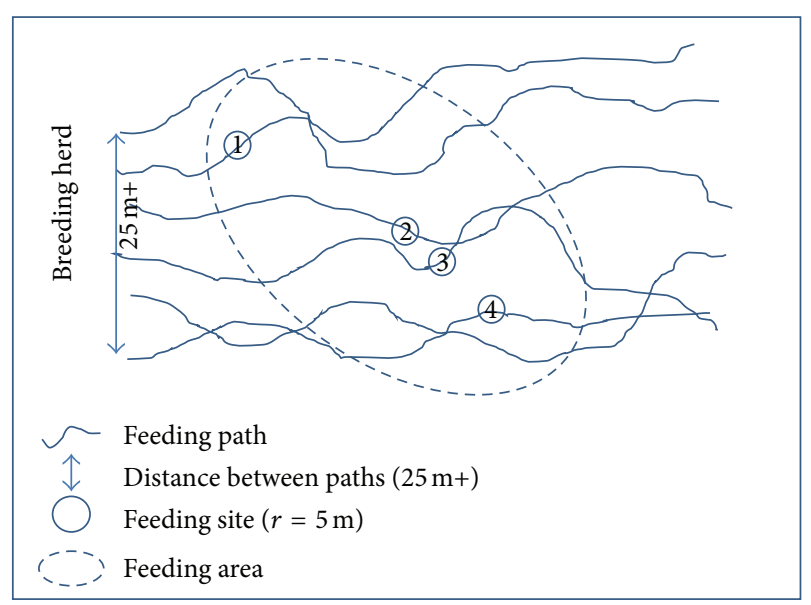

FIGURE 1: A diagram illustrating sampling methodology, that is, feeding paths, feeding area and feeding site.

data of all-male groups or solitary elephant bulls, as sexual dimorphism affects their feeding behaviour [26].

2.3. Data Collection. We collected data during two wet seasons and two dry seasons in the early and late phases of each season, commencing in March 2002 and ending in September 2005. Two criteria were used to define a feeding area prior to the recording of floristic data (Figure 1).

Firstly, average feeding time measured among at least three individuals in the breeding herd had to exceed $1.5 \mathrm{~min}$. Secondly, the distance between feeding paths within a feeding area had to exceed $25 \mathrm{~m}$. Individual elephants in breeding herds tend to move closer together as a unit when not feeding and especially when crossing open areas or when they perceive imminent danger (J. J. Viljoen, H. C. Reynecke, pers. obs.). Recording data during real-time observations following these criteria eliminated suppositions of the source and nature of the impact due to, that is, chance, accidental, fire, frost, droughts, natural mortality, other herbivores, or elephant bulls [27]. We then identified, using current field guides [28], all woody plants, available to elephants in feeding sites and recorded them as either utilised or notutilised. Feeding sites had a radius of $5 \mathrm{~m}\left(\right.$ area $\left.=78.5 \mathrm{~m}^{2}\right)$ measured from the sighting of the first elephant from the herd feeding, assuming that this area would contain the impact of an individual $[6,26]$.

Once data had been collected at the feeding site observed to have been browsed, an alternative feeding path in the feeding area was followed to limit the chance of collecting data from the same individual's selection. We collected data from four feeding sites selected in the feeding area occupied by a herd to allow for a better representation of the woody species present in the area. Cerling et al. [29] reported that isotope patterns in hairs of different individuals from the same elephant herd were similar which represented dietary preferences and behaviour of the entire group. Similarly, Buss [18] concluded that food habits and other behaviour of individuals within each group were similar based on 


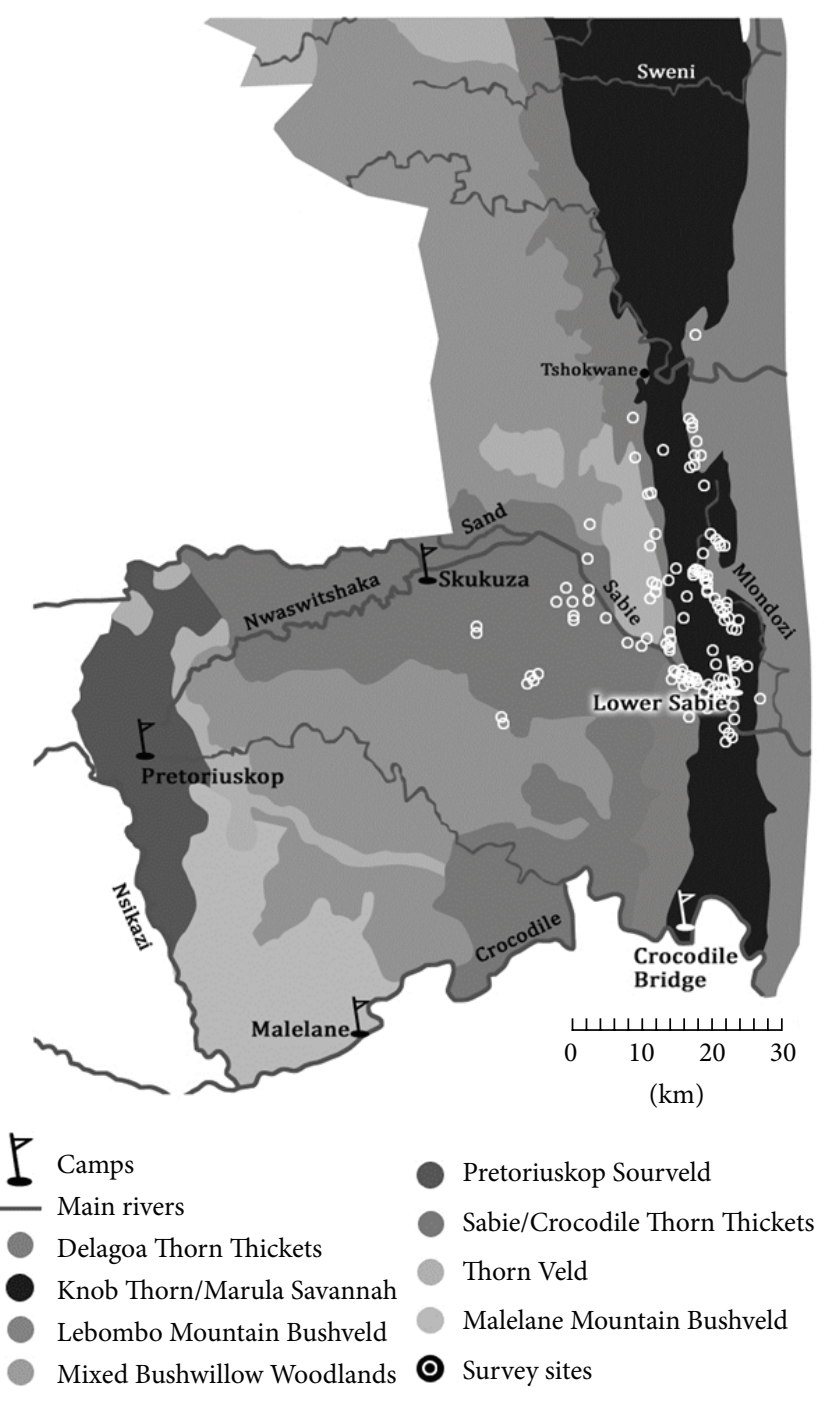

(a)

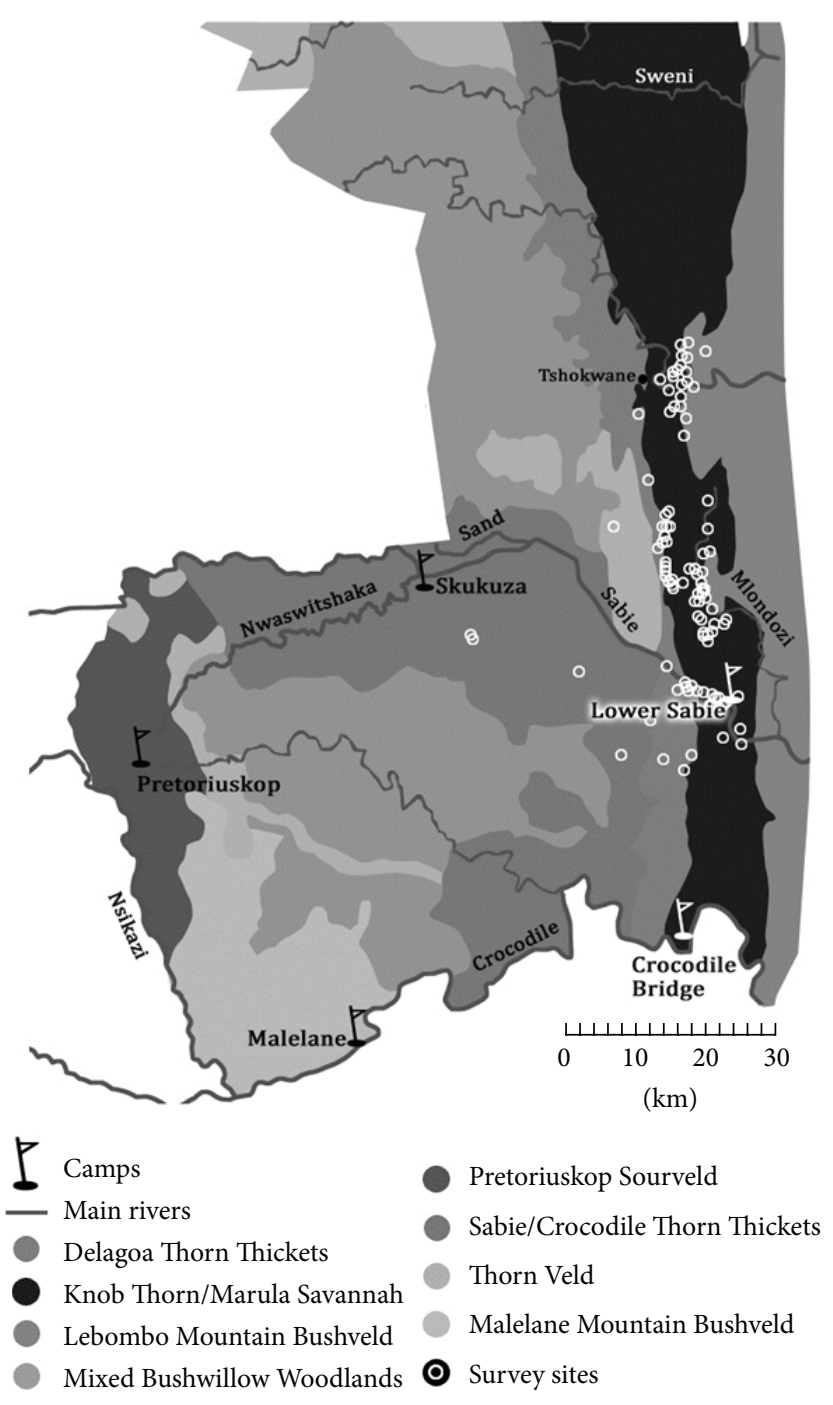

(b)

FIGURE 2: The movements of the breeding herds of elephants and location of the survey sites during the wet (a) and dry (b) seasons in the southern Kruger National Park from March 2002 to September 2005. (ecozones ex, Gertenbach [17]).

field observations on feeding behaviour of elephants on the savanna lands in the Murchison Falls National Park region of Uganda.

Field data recording commenced anywhere between 10 and $60 \mathrm{~min}$ after a breeding herd had left the observed feeding area. Field crews (at least two observers) maintained a safe distance from the animals to minimize disturbance and always avoided being positioned upwind of the herd to avoid detection. Direct field observations in Uganda on feeding behaviour of elephants indicated that the primary feeding periods occurred most frequently during early morning and only minor or secondary quantities of food were selected at various times of day and night which supplemented their diets [18].

Each elephant within the breeding herd left behind numerous feeding sites on the feeding path, while moving slowly through the feeding area before regrouping and moving towards the next feeding area or to water.
2.4. Data Analysis. An animal which has access to a variety of food items will show preference for some and avoid others [30]. Preference was measured by comparing selection of woody species in proportion to their estimated availability. Seasonal preferences for woody species were then ranked according to Manly's alpha $(\alpha)$ index of preference [30]:

$$
\alpha_{i}=\frac{r_{i}}{n_{i}}\left(\frac{1}{\sum r_{j} / n_{j}}\right)
$$

Where $\alpha_{i}$ is Manly's $\propto$ (preference index) for woody species $i, r_{i}, r_{j}$ is the proportion of woody species $i$ or $j$ selected ( $i$ and $j=1,2,3 \ldots, m), n_{i}, n_{j}$ is the proportion of woody species $i$ or $j$ in the environment, and $m$ is the total number of tree species available.

If the index value $\alpha_{i}>1 / m$, then the woody species $i$ is given preference for selection, whereas if $\alpha_{i}<1 / m$, the woody species $i$ is neglected. 
TABLE 1: Manly's alpha index values of species selected (S) and neglected (N) in the early November-January and late wet seasons (FebruaryApril) by African elephant breeding herds in the southern Kruger National Park from March 2002 to September 2005. If $\alpha>1 / m$, then the species is considered to be selected, while if $\alpha<1 / m$, the species is neglected.

\begin{tabular}{|c|c|c|c|c|c|}
\hline \multicolumn{3}{|c|}{ Early wet season $(1 / m=0.0227)$} & \multicolumn{3}{|c|}{ Late wet season $(1 / m=0.0263)$} \\
\hline Species & $\alpha$ & Selected/neglected & Species & $\alpha$ & Selected/neglected \\
\hline Manilkara mochisia & 0.0866 & $\mathrm{~S}$ & Vachellia nilotica & 0.0963 & S \\
\hline Schotia capitata & 0.0866 & $\mathrm{~S}$ & Boscia mossambicensis & 0.0963 & S \\
\hline Strychnos madagascariensis & 0.0866 & $\mathrm{~S}$ & Ozoroa obovata & 0.0723 & S \\
\hline Bolusanthus speciosus & 0.0577 & $\mathrm{~S}$ & Clerodendrum glabrum & 0.0642 & S \\
\hline Peltophorum africanum & 0.0469 & $S$ & Albizia harveyi & 0.0482 & S \\
\hline Carissa bispinosa & 0.0433 & $\mathrm{~S}$ & Sclerocarya birrea & 0.0482 & S \\
\hline Combretum molle & 0.0433 & S & Ximenia americana & 0.0482 & S \\
\hline Grewia hexamita & 0.0408 & S & Grewia bicolor & 0.0351 & S \\
\hline Boscia albitrunca & 0.0371 & S & Grewia monticola & 0.0341 & S \\
\hline Sclerocarya birrea & 0.0371 & S & Boscia albitrunca & 0.0321 & $S$ \\
\hline Lannea schweinfurthii & 0.0334 & $\mathrm{~S}$ & Searsia gueinzii & 0.0321 & S \\
\hline Albizia petersiana & 0.0289 & $\mathrm{~S}$ & Strychnos spinosa & 0.0321 & S \\
\hline Dombeya rotundifolia & 0.0289 & $S$ & Lannea schweinfurthii & 0.0321 & $S$ \\
\hline Ozoroa engleri & 0.0266 & $\mathrm{~S}$ & Grewia flavescens & 0.0299 & S \\
\hline Cassia abbreviata & 0.0217 & $\mathrm{~N}$ & Grewia hexamita & 0.0282 & S \\
\hline Mundulea sericea & 0.0217 & $\mathrm{~N}$ & Manilkara mochisia & 0.0241 & $\mathrm{~N}$ \\
\hline Cissus cornifolia & 0.0204 & $\mathrm{~N}$ & Terminalia prunioides & 0.0222 & $\mathrm{~N}$ \\
\hline Diospyros mespiliformis & 0.0200 & $\mathrm{~N}$ & Combretum apiculatum & 0.0218 & $\mathrm{~N}$ \\
\hline Grewia bicolor & 0.0192 & $\mathrm{~N}$ & Combretum zeyheri & 0.0201 & $\mathrm{~N}$ \\
\hline Grewia flavescens & 0.0164 & $\mathrm{~N}$ & Ziziphus mucronata & 0.0186 & $\mathrm{~N}$ \\
\hline Searsia gueinzii & 0.0162 & $\mathrm{~N}$ & Combretum imberbe & 0.0161 & $\mathrm{~N}$ \\
\hline Pterocarpus rotundifolius & 0.0156 & $\mathrm{~N}$ & Albizia forbesii & 0.0140 & $\mathrm{~N}$ \\
\hline Ximenia caffra & 0.0144 & $\mathrm{~N}$ & Dalbergia melanoxylon & 0.0139 & $\mathrm{~N}$ \\
\hline Euclea divinorum & 0.0131 & $\mathrm{~N}$ & Pterocarpus rotundifolius & 0.0129 & $\mathrm{~N}$ \\
\hline Albizia forbesii & 0.0124 & $\mathrm{~N}$ & Diospyros mespiliformis & 0.0126 & $\mathrm{~N}$ \\
\hline Combretum zeyheri & 0.0124 & $\mathrm{~N}$ & Euclea divinorum & 0.0114 & $\mathrm{~N}$ \\
\hline Dichrostachys cinerea & 0.0118 & $\mathrm{~N}$ & Vachellia tortilis & 0.0113 & $\mathrm{~N}$ \\
\hline Ehretia amoena & 0.01100 & $\mathrm{~N}$ & Ehretia amoena & 0.0098 & $\mathrm{~N}$ \\
\hline Dalbergia melanoxylon & 0.0107 & $\mathrm{~N}$ & Gymnosporia buxifolia & 0.0092 & $\mathrm{~N}$ \\
\hline Vachellia nigrescens & 0.0105 & $\mathrm{~N}$ & Gymnosporia senegalensis & 0.0089 & $\mathrm{~N}$ \\
\hline Flueggea virosa & 0.0084 & $\mathrm{~N}$ & Dichrostachys cinerea & 0.0084 & $\mathrm{~N}$ \\
\hline Vachellia robusta & 0.0081 & $\mathrm{~N}$ & Combretum hereroense & 0.0075 & $\mathrm{~N}$ \\
\hline Combretum collinum & 0.0072 & $\mathrm{~N}$ & Vachellia robusta & 0.0064 & $\mathrm{~N}$ \\
\hline Combretum hereroense & 0.0069 & $\mathrm{~N}$ & Vachellia nigrescens & 0.0057 & $\mathrm{~N}$ \\
\hline Ziziphus mucronata & 0.0064 & $\mathrm{~N}$ & Philenoptera violacea & 0.0055 & $\mathrm{~N}$ \\
\hline Vachellia gerrardii & 0.0054 & $\mathrm{~N}$ & Combretum paniculatum & 0.0044 & $\mathrm{~N}$ \\
\hline Gymnosporia senegalensis & 0.0052 & $\mathrm{~N}$ & Grewia villosa & 0.0040 & $\mathrm{~N}$ \\
\hline Combretum paniculatum & 0.0048 & $\mathrm{~N}$ & Flueggea virosa & 0.0019 & $\mathrm{~N}$ \\
\hline Grewia villosa & 0.0040 & $\mathrm{~N}$ & & & \\
\hline Vachellia tortilis & 0.0036 & $\mathrm{~N}$ & & & \\
\hline Combretum apiculatum & 0.0029 & $\mathrm{~N}$ & & & \\
\hline Euclea undulate & 0.0024 & $\mathrm{~N}$ & & & \\
\hline Philenoptera violacea & 0.0022 & $\mathrm{~N}$ & & & \\
\hline Cienfuegosia hildebrandtii & 0.0012 & $\mathrm{~N}$ & & & \\
\hline
\end{tabular}


TABLE 2: Manly's alpha index values of species selected (S) and neglected (N) in the early May-July and late dry seasons (August-October) by African elephant breeding herds in the southern Kruger National Park from March 2002 to September 2005. If $\alpha>1 / m$, then the species is considered to be selected, while if $\alpha<1 / m$, the species is neglected.

\begin{tabular}{|c|c|c|c|c|c|}
\hline \multicolumn{3}{|c|}{ Early dry season $(1 / m=0.0161)$} & \multicolumn{3}{|c|}{ Late dry season $(1 / m=0.0145)$} \\
\hline Species & $\alpha$ & Selected/neglected & Species & $\alpha$ & Selected/neglected \\
\hline Manilkara mochisia & 0.093736 & $S$ & Boscia mossambicensis & 1 & $S$ \\
\hline Searsia gueinzii & 0.065615 & S & Commiphora mollis & 1 & S \\
\hline Grewia monticola & 0.06249 & S & Croton megalobotrys & 1 & S \\
\hline Vachellia xanthophloea & 0.056241 & S & Grewia occidentalis & 1 & S \\
\hline Grewia bicolor & 0.04838 & S & Peltophorum africanum & 0.9286 & S \\
\hline Ozoroa engleri & 0.046868 & S & Vachellia xanthophloea & 0.8571 & S \\
\hline Rhoicissus spp. & 0.046868 & S & Schotia brachypetala & 0.5714 & S \\
\hline Terminalia sericea & 0.043263 & S & Grewia monticola & 0.5616 & S \\
\hline Ziziphus mucronata & 0.04166 & S & Euclea natalensis & 0.5 & S \\
\hline Vachellia gerrardii & 0.040534 & S & Kigelia africana & 0.5 & S \\
\hline Combretum imberbe & 0.037494 & S & Spirostachys africana & 0.5 & S \\
\hline Grewia hexamita & 0.036896 & S & Terminalia sericea & 0.5 & S \\
\hline Dombeya rotundifolia & 0.035151 & S & Grewia bicolor & 0.4901 & S \\
\hline Peltophorum africanum & 0.031245 & S & Grewia hexamita & 0.4419 & S \\
\hline Schotia brachypetala & 0.031245 & S & Vachellia gerrardii & 0.4 & S \\
\hline Lannea schweinfurthii & 0.027269 & S & Combretum imberbe & 0.3636 & S \\
\hline Pterocarpus rotundifolius & 0.027102 & S & Albizia forbesii & 0.2941 & S \\
\hline Vachellia nigrescens & 0.024996 & S & Grewia villosa & 0.2895 & $S$ \\
\hline Albizia petersiana & 0.022318 & S & Combretum zeyheri & 0.2857 & S \\
\hline Vachellia robusta & 0.020377 & S & Dalbergia melanoxylon & 0.2787 & S \\
\hline Diospyros mespiliformis & 0.019228 & S & Vachellia tortilis & 0.25 & S \\
\hline Ozoroa obovata & 0.018747 & S & Manilkara mochisia & 0.25 & $S$ \\
\hline Euclea divinorum & 0.017439 & S & Mundulea sericea & 0.25 & S \\
\hline Combretum hereroense & 0.017267 & S & Searsia gueinzii & 0.25 & $S$ \\
\hline Dalbergia melanoxylon & 0.015539 & $\mathrm{~N}$ & Diospyros mespiliformis & 0.2143 & S \\
\hline Albizia forbesii & 0.013339 & $\mathrm{~N}$ & Euclea divinorum & 0.1912 & S \\
\hline Grewia flavescens & 0.012226 & $\mathrm{~N}$ & Combretum paniculatum & 0.1818 & S \\
\hline Sclerocarya birrea & 0.010415 & $\mathrm{~N}$ & Ochna inermis & 0.1667 & S \\
\hline Vachellia tortilis & 0.008521 & $\mathrm{~N}$ & Vachellia nigrescens & 0.1386 & $S$ \\
\hline Dichrostachys cinerea & 0.008497 & $\mathrm{~N}$ & Ozoroa engleri & 0.125 & S \\
\hline Ximenia caffra & 0.006695 & $\mathrm{~N}$ & Grewia flavescens & 0.1134 & S \\
\hline Gymnosporia senegalensis & 0.006465 & $\mathrm{~N}$ & Vachellia robusta & 0.1132 & $S$ \\
\hline Grewia villosa & 0.003125 & $\mathrm{~N}$ & Commiphora pyracanthoides & 0.1111 & S \\
\hline Flueggea virosa & 0.001644 & $\mathrm{~N}$ & Gymnosporia senegalensis & 0.1111 & S \\
\hline Ehretia amoena & 0.001103 & $\mathrm{~N}$ & Vachellia erubescens & 0.1 & S \\
\hline Vachellia erubescens & 0 & $\mathrm{~N}$ & Pterocarpus rotundifolius & 0.0976 & $S$ \\
\hline Vachellia exuvialis & 0 & $\mathrm{~N}$ & Vachellia exuvialis & 0.087 & S \\
\hline Bolusanthus speciosus & 0 & $\mathrm{~N}$ & Dichrostachys cinerea & 0.07 & S \\
\hline Boscia foetida & 0 & $\mathrm{~N}$ & Ziziphus mucronata & 0.0455 & S \\
\hline Cissus cornifolia & 0 & $\mathrm{~N}$ & Ehretia amoena & 0.0323 & S \\
\hline Combretum apiculatum & 0 & $\mathrm{~N}$ & Combretum apiculatum & 0.0313 & $S$ \\
\hline Commiphora mollis & 0 & $\mathrm{~N}$ & Combretum hereroense & 0.0238 & S \\
\hline Commiphora pyracanthoides & 0 & $\mathrm{~N}$ & Boscia foetida & 0.0213 & S \\
\hline Croton megalobotrys & 0 & $\mathrm{~N}$ & Vachellia grandicornuta & 0 & $\mathrm{~N}$ \\
\hline Ehretia rigida & 0 & $\mathrm{~N}$ & Albizia petersiana & 0 & $\mathrm{~N}$ \\
\hline Euclea natalensis & 0 & $\mathrm{~N}$ & Balanites maughamii & 0 & $\mathrm{~N}$ \\
\hline Euphorbia ingens & 0 & $\mathrm{~N}$ & Berchemia discolor & 0 & $\mathrm{~N}$ \\
\hline Grewia occidentalis & 0 & $\mathrm{~N}$ & Bolusanthus speciosus & 0 & $\mathrm{~N}$ \\
\hline
\end{tabular}


TABLE 2: Continued.

\begin{tabular}{|c|c|c|c|c|c|}
\hline \multicolumn{3}{|c|}{ Early dry season $(1 / m=0.0161)$} & \multicolumn{3}{|c|}{ Late dry season $(1 / m=0.0145)$} \\
\hline Species & $\alpha$ & Selected/neglected & Species & $\alpha$ & Selected/neglected \\
\hline Gymnosporia buxifolia & 0 & $\mathrm{~N}$ & Boscia albitrunca & 0 & $\mathrm{~N}$ \\
\hline Gymnosporia maranguensis & 0 & $\mathrm{~N}$ & Carissa bispinosa & 0 & $\mathrm{~N}$ \\
\hline Hyperacanthus amoenus & 0 & $\mathrm{~N}$ & Commiphora africana & 0 & $\mathrm{~N}$ \\
\hline Oncoba spinosa & 0 & $\mathrm{~N}$ & Ehretia rigida & 0 & $\mathrm{~N}$ \\
\hline Ormocarpum trichocarpum & 0 & $\mathrm{~N}$ & Euclea undulata & 0 & $\mathrm{~N}$ \\
\hline Philenoptera violacea & 0 & $\mathrm{~N}$ & Flueggea virosa & 0 & $\mathrm{~N}$ \\
\hline Phoenix reclinata & 0 & $\mathrm{~N}$ & Gardenia volkensii & 0 & $\mathrm{~N}$ \\
\hline Phyllanthus reticulatus & 0 & $\mathrm{~N}$ & Gymnosporia buxifolia & 0 & $\mathrm{~N}$ \\
\hline Senna petersiana & 0 & $\mathrm{~N}$ & Lannea schweinfurthii & 0 & $\mathrm{~N}$ \\
\hline Spirostachys africana & 0 & $\mathrm{~N}$ & Mystroxylon aethiopicum & 0 & $\mathrm{~N}$ \\
\hline Strychnos madagascariensis & 0 & $\mathrm{~N}$ & Oncoba spinosa & 0 & $\mathrm{~N}$ \\
\hline Terminalia phanerophlebia & 0 & $\mathrm{~N}$ & Ormocarpum trichocarpum & 0 & $\mathrm{~N}$ \\
\hline Terminalia prunioides & 0 & $\mathrm{~N}$ & Philenoptera violacea & 0 & $\mathrm{~N}$ \\
\hline \multirow[t]{8}{*}{ Ximenia americana } & 0 & $\mathrm{~N}$ & Phyllanthus reticulatus & 0 & $\mathrm{~N}$ \\
\hline & & & Ptaeroxylon obliquum & 0 & $\mathrm{~N}$ \\
\hline & & & Schotia caffra & 0 & $\mathrm{~N}$ \\
\hline & & & Sclerocarya birrea & 0 & $\mathrm{~N}$ \\
\hline & & & Senna petersiana & 0 & $\mathrm{~N}$ \\
\hline & & & Strychnos spinosa & 0 & $\mathrm{~N}$ \\
\hline & & & Terminalia phanerophlebia & 0 & $\mathrm{~N}$ \\
\hline & & & Ximenia americana & 0 & $\mathrm{~N}$ \\
\hline
\end{tabular}

Data recorded from the woody plant species utilised and not utilised from the collared and noncollared herds were collated according to season. Availability and selection of woody species in the sample sites for all seasons were categorised with a frequency distribution into classes. A woody species with an observed frequency between 0 and 100 in all feeding sites was categorised as rare and with a frequency $>100$ as common. Similarly, woody species with a selection frequency between 0 and 25 were categorised as low selection and $>25$ as high selection. Statistica version 7 was used for all analyses [31].

\section{Results}

Average annual rainfall for the Lower Sabie area during the study period measured $549 \mathrm{~mm}$ during the wet seasons and $25 \mathrm{~mm}$ for the dry seasons. Areas traversed by observed herds during the study period extended from the Sabie and Crocodile River Thickets, Delagoa Thorn Thickets, Sclerocarya birrea subsp. caffra, Vachellia nigrescens Savanna, Mixed Combretum Woodlands to the Lebombo Mountain Bushveld. We sampled 237 feeding areas which included 468 wet season feeding sites and 480 dry season feeding sites (Figure 2). We recorded 98 woody species within the feeding areas and of this number; 63 species contributed to the elephant's diet across all seasons.

Based on average percentages of the Manly alpha index values, the woody plants neglected decreased from the wet seasons $(65 \%)$ to the dry season (41\%) (Figure 3 ). This is confirmed by the values $<0.1$ for woody plants in the early wet, late wet, and early dry seasons indicating a low proportion of woody plants being selected (Tables 1 and 2). During the late dry seasons 34 woody plant species had values ranging from 0.1 to 1.0 (Table 2). This change towards a greater proportion of woody species recorded in the dry seasons indicates that the herds selected different vegetation types in the dry season compared to wet seasons. This change might be from the low grass production during the dry season and availability of a diversity of woody plant species in southern KNP [32].

Three Grewia species, namely, Grewia bicolor, Grewia flavescens, and Grewia monticola which have a wide distribution in the landscape, were selected in all seasons except in the early wet season, and Grewia hexamita was the only woody plant recorded as being highly selected in all four seasons (Table 3). van Wyk and Fairall [33] reported that Grewia species were sought after by elephants in the southern region of the KNP. Rarity of occurrence, that is, species occurring at low frequencies (less than 100 individuals in all feeding sites), did not stop elephants from actively seeking out these plants. Of the 63 plants selected across all seasons, only 10 plants were common, and 53 plants were classified as rare (Table 3 ).

\section{Discussion}

Although it is reported that elephants have a preference for the riparian fringe [34] and are frequently associated with that plant community (Figure 2), our results suggest that they do not have any feeding preference for woody 
TABLE 3: Woody species selected (high (h), low (l)) and available (rare (r), common (c)) in the feeding areas during different seasons frequented by African elephant breeding herds in the southern Kruger National Park from March 2002 to September 2005.

\begin{tabular}{|c|c|c|c|c|}
\hline Species & Early wet & Late wet & Early dry & Late dry \\
\hline Grewia hexamita & $h, r$ & $h, r$ & $h, r$ & $h, r$ \\
\hline Grewia bicolor & & h, c & h, c & h, c \\
\hline Grewia flavescens & & h, c & $1, \mathrm{r}$ & $1, \mathrm{r}$ \\
\hline Grewia monticola & & h, c & $h, r$ & $\mathrm{~h}, \mathrm{r}$ \\
\hline Searsia gueinzii & & $\mathrm{h}, \mathrm{r}$ & $h, r$ & $h, r$ \\
\hline Manilkara mochisia & $\mathrm{h}, \mathrm{r}$ & & $h, r$ & $h, r$ \\
\hline Ozoroa engleri & $h, r$ & & $h, r$ & $1, \mathrm{r}$ \\
\hline Peltophorum africanum & $h, r$ & & $h, r$ & $h, r$ \\
\hline Schotia brachypetala & $h, r$ & & $h, r$ & $h, r$ \\
\hline Lannea schweinfurthii & $1, \mathrm{r}$ & $h, r$ & $h, r$ & \\
\hline Sclerocarya birrea & $1, \mathrm{r}$ & $h, r$ & $1, \mathrm{r}$ & \\
\hline Vachellia gerrardii & & & $h, r$ & $h, r$ \\
\hline Vachellia nigrescens & & & $h, r$ & $1, \mathrm{c}$ \\
\hline Vachellia robusta & & & $1, \mathrm{r}$ & $1, \mathrm{r}$ \\
\hline Vachellia tortilis & & & $1, \mathrm{r}$ & h, c \\
\hline Vachellia xanthophloea & & & $h, r$ & $h, r$ \\
\hline Albizia forbesii & & & $1, \mathrm{c}$ & $h, r$ \\
\hline Combretum hereroense & & & $1, \mathrm{r}$ & $1, \mathrm{r}$ \\
\hline Combretum imberbe & & & $h, r$ & $h, r$ \\
\hline Dalbergia melanoxylon & & & $1, \mathrm{c}$ & $h, r$ \\
\hline Dichrostachys cinerea & & & $1, \mathrm{c}$ & $1, \mathrm{c}$ \\
\hline Diospyros mespiliformis & & & $1, \mathrm{r}$ & $1, \mathrm{r}$ \\
\hline Euclea divinorum & & & $1, \mathrm{r}$ & $1, \mathrm{c}$ \\
\hline Grewia villosa & & & $1, \mathrm{r}$ & $h, r$ \\
\hline Gymnosporia senegalensis & & & $1, \mathrm{c}$ & l, c \\
\hline Pterocarpus rotundifolius & & & h, c & $1, \mathrm{r}$ \\
\hline Terminalia sericea & & & $h, r$ & $h, r$ \\
\hline Ziziphus mucronata & & & $h, r$ & $1, \mathrm{r}$ \\
\hline Boscia mossambicensis & & $h, r$ & & $h, r$ \\
\hline Ozoroa obovata & & $h, r$ & $1, \mathrm{r}$ & \\
\hline Albizia petersiana & $h, r$ & & $1, \mathrm{r}$ & \\
\hline Dombeya rotundifolia & $h, r$ & & $h, r$ & \\
\hline Boscia albitrunca & $h, r$ & h, r & & \\
\hline Vachellia erubescens & & & & $1, \mathrm{r}$ \\
\hline Vachellia exuvialis & & & & $1, \mathrm{r}$ \\
\hline Boscia foetida & & & & $1, \mathrm{r}$ \\
\hline Combretum apiculatum & & & & $1, \mathrm{r}$ \\
\hline Combretum paniculatum & & & & $1, \mathrm{r}$ \\
\hline Combretum zeyheri & & & & $h, r$ \\
\hline Commiphora mollis & & & & $h, r$ \\
\hline Commiphora pyracanthoides & & & & $1, \mathrm{r}$ \\
\hline Croton megalobotrys & & & & $h, r$ \\
\hline Ehretia amoena & & & & $1, \mathrm{r}$ \\
\hline Euclea natalensis & & & & $1, \mathrm{r}$ \\
\hline Grewia occidentalis & & & & $h, r$ \\
\hline Kigelia africana & & & & $\mathrm{h}, \mathrm{r}$ \\
\hline Mundulea sericea & & & & $h, r$ \\
\hline Ochna inermis & & & & $1, \mathrm{r}$ \\
\hline Spirostachys africana & & & & $1, \mathrm{r}$ \\
\hline Ziziphus mucronata & & & & $1, \mathrm{r}$ \\
\hline
\end{tabular}


TABle 3: Continued.

\begin{tabular}{lccc}
\hline Species & Early wet & Late wet & Early dry \\
\hline $\begin{array}{l}\text { Terminalia sericea } \\
\text { Flueggea virosa }\end{array}$ & & & Late dry \\
$\begin{array}{l}\text { Rhoicissus spp. } \\
\text { Ximenia caffra }\end{array}$ & & & $\mathrm{h}, \mathrm{r}$ \\
Vachellia nilotica & & $\mathrm{h}, \mathrm{r}$ & $\mathrm{r}$ \\
Albizia harveyi & & $\mathrm{h}, \mathrm{r}$ & $\mathrm{h}, \mathrm{r}$ \\
Clerodendrumglabrum & & $\mathrm{h}, \mathrm{r}$ & \\
Strychnos spinosa & & $\mathrm{h}, \mathrm{r}$ & \\
Ximenia americana & $\mathrm{h}, \mathrm{r}$ & & \\
Bolusanthus speciosus & $\mathrm{h}, \mathrm{r}$ & & \\
Carissa bispinosa & $\mathrm{h}, \mathrm{r}$ & $\mathrm{h}, \mathrm{r}$ & \\
Combretum molle & & & \\
Schotia capitata & & \\
\hline
\end{tabular}

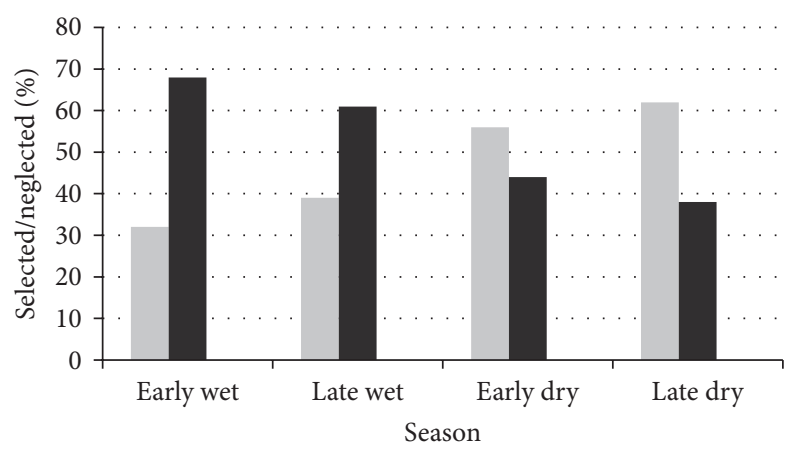

Selected \%

Neglected \%

FIGURE 3: Percentage of woody species selected and neglected, based on Manly's alpha values, in the feeding areas selected by breeding herds of African elephants from March 2002 to September 2005 in the southern Kruger National Park.

plants in the riparian fringe. Tree species generally associated with riparian vegetation such as Ficus sycomorus, Breonadia salicina, and Nuxia oppositifolia are commonly found along rivers in the study area. However, the feeding sites actively selected by breeding herds in our study did not contain these woody species. Thus, the association of elephants with riparian fringes might rather be for regulating body temperature and feeding on the reed Phragmites australis in the riverbeds (J. J. Viljoen, H.C. Reynecke, pers. obs.).

As there are insufficient quantitative data available, no clear prediction has emerged about which woody species are prone to extirpation and under what circumstances [35]. Kerley et al. [12] made reference to the lack of quantitative studies in terms of species contribution in diet of elephants as well as the plethora of studies that indirectly infer elephant diet from plant-based studies. For example, Sclerocarya birrea is a woody plant that is deemed a species susceptible to extirpation $[35,36]$; yet, our data show that this plant had a high selection frequency only in the late wet season at a low Manly alpha index value of 0.048 . Although present in the feeding areas in the early dry and late dry seasons, this plant was neglected by the breeding herds of elephants.

To a large degree climatic and biological variability in African savannas explain differences in heterogeneity and productivity of vegetation at landscape and regional scales $[14,35]$. These explain the differential use of habitats and seasonal changes in the distribution of elephants [7, 37-44]. This study shows that the selection preference of elephant herds corresponds with seasonal changes [29, 44]. In the feeding areas frequented by the observed elephant breeding herds, $64 \%$ of the available woody species were browsed by elephants during the four seasons. This relatively high proportion of sought after woody species suggests that breeding herds select areas that permit efficient and effective nutrient intake $[29,45]$. Functional traits of selected woody species, that is, their phenology and life history, probably vary seasonally, which could explain these selection patterns. Future research should investigate the causal mechanisms underlying selection preferences, which might include plant nutrients in the soil, leaves, fruits, and flowers of woody species.

Although our observations were restricted to diurnal hours due to safety issues and visibility, we propose that the woody plant species available and selected during the night did not differ from those selected during daytime [18].

\section{References}

[1] G. I. H. Kerley and M. Landman, "The impacts of elephants on biodiversity in the Eastern Cape Subtropical Thickets," South African Journal of Science, vol. 102, no. 9-10, pp. 395-402, 2006.

[2] K. A. Y. Hiscocks, "The impact of an increasing elephant population on the woody vegetation in southern Sabi Sand Wildtuin, South Africa," Koedoe, vol. 42, no. 2, pp. 47-55, 1999.

[3] R. J. van Aarde, T. P. Jackson, and S. M. Ferreira, "Conservation science and elephant management in Southern Africa," South African Journal of Science, vol. 102, no. 9-10, pp. 385-388, 2006.

[4] Garaï, M. E, R. Slotow, R. D. Carr, and B. Reilly, "Elephant reintroductions to small fenced reserves in South Africa," Pachyderm, vol. 37, pp. 28-36, 2004. 
[5] R. Slotow, M. E. Garaï, B. Reilly, B. Page, and R. D. Carr, "Population dynamics of elephants re-introduced to small fenced reserves in South Africa," South African Journal of Wildlife Research, vol. 35, no. 1, pp. 23-32, 2005.

[6] G. D. Anderson and B. H. Walker, "Vegetation composition and elephant damage in the Sengwa wildlife research area, Rhodesia," Journal of the South African Wildlife Management Association, vol. 4, no. 1, pp. 1-14, 1974.

[7] D. Western, "Water availability and its influences on the structure and dynamics of a savannah large community," African Journal of Ecology, vol. 13, no. 3-4, pp. 265-288, 1975.

[8] Y. de Beer, W. Kilian, W. Versfeld, and R. J. van Aarde, "Elephants and low rainfall alter woody vegetation in Etosha National Park, Namibia," Journal of Arid Environments, vol. 64, no. 3, pp. 412-421, 2006.

[9] R. M. Holdo, "Elephants, fire, and frost can determine community structure and composition in Kalahari woodlands," Ecological Applications, vol. 17, no. 2, pp. 558-568, 2007.

[10] P. W. J. Baxter and W. M. Getz, "Development and parameterization of a rain- and fire-driven model for exploring elephant effects in African savannas," Environmental Modeling and Assessment, vol. 13, no. 2, pp. 221-242, 2008.

[11] J. Chafota and N. Owen-Smith, "Episodic severe damage to canopy trees by elephants: Interactions with fire, frost and rain," Journal of Tropical Ecology, vol. 25, no. 3, pp. 341-345, 2009.

[12] G. I. H. Kerley, M. Landman, L. Kruger, and N. OwenSmith, "Effects of elephants on ecosystems and biodiversity," in Elephant Management, R. J. Scholes and K. G. Mennell, Eds., chapter 3, pp. 146-205, Wits University Press, Johannesburg, South Africa, 2008.

[13] P. F. Scogings, R. W. Taylor, and D. Ward, "Inter- and intra-plant variations in nitrogen, tannins and shoot growth of Sclerocarya birrea browsed by elephants," Plant Ecology, vol. 213, no. 3, pp. 483-491, 2012.

[14] N. Owen-Smith, G. I. H. Kerley, B. Page, R. Slotow, and R. J. van Aarde, "A scientific perspective on the management of elephants in the Kruger National Park and elsewhere," South African Journal of Science, vol. 102, no. 9-10, pp. 389-394, 2006.

[15] A. M. White and P. S. Goodman, "Differences in woody vegetation are unrelated to use by African elephants (Loxodonta africana) in Mkhuze Game Reserve, South Africa," African Journal of Ecology, vol. 48, no. 1, pp. 215-223, 2010.

[16] M. Landman, G. I. H. Kerley, and D. S. Schoeman, "Relevance of elephant herbivory as a threat to important plants in the addo elephant National Park, South Africa," Journal of Zoology, vol. 274, no. 1, pp. 51-58, 2008.

[17] W. P. D. Gertenbach, "Landscapes of the Kruger National Park," Koedoe, vol. 26, pp. 9-121, 1983.

[18] I. O. Buss, "Some observations on food habits and behavior of the African elephant," The Journal of Wildlife Management, vol. 25, no. 2, pp. 131-148, 1961.

[19] F. V. Osborn, "The concept of home range in relation to elephants in Africa," Pachyderm, vol. 37, pp. 37-44, 2004.

[20] J. Codron, D. Codron, J. A. Lee-Thorp et al., "Landscapescale feeding patterns of African elephant inferred from carbon isotope analysis of feces," Oecologia, vol. 165, no. 1, pp. 89-99, 2011.

[21] L. Mucina and M. C. Rutherford, Eds., The Vegetation of South Africa, Lesotho and Swaziland, vol. 19 of Sterlizia, South African National Biodiversity Institute, Pretoria, South Africa, 2006.
[22] F. J. Venter, "Soil patterns associated with the major geological units of the Kruger National Park," Koedoe, vol. 29, pp. 125-138, 1986.

[23] F. J. Venter, R. J. Scholes, and H. C. Eckhardt, "The abiotic template and its associated vegetation pattern," in The Kruger Experience, J. T. Du Toit, K. H. Rogers, and H. C. Biggs, Eds., chapter 5, pp. 83-129, Island Press, Washington, DC, USA, 2003.

[24] N. Zambatis, "Annual section ranger reports. KNP vegetation monitoring," Scientific Services - Kruger National Park. In press.

[25] E. A. Archie, C. J. Moss, and S. C. Alberts, "The ties that bind: genetic relatedness predicts the fission and fusion of social groups in wild African elephants," Proceedings of the Royal Society B, vol. 273, no. 1586, pp. 513-522, 2006.

[26] S. Stokke and J. T. Du Toit, "Sex and size related differences in the dry season feeding patterns of elephants in Chobe National Park, Botswana," Ecography, vol. 23, no. 1, pp. 70-80, 2000.

[27] R. I. Yeaton, "Porcupines, fires and the dynamics of the tree layer of the Burkea africana savanna," Journal of Ecology, vol. 76, no. 4, pp. 1017-1029, 1988.

[28] B. van Wyk and P. van Wyk, Field Guide to the Trees of Southern Africa, Field Guides Series, Struik Publishers, Cape Town, South Africa, 1997.

[29] T. E. Cerling, B. H. Passey, L. K. Ayliffe et al., “Orphans' tales: seasonal dietary changes in elephants from Tsavo National Park, Kenya," Palaeogeography, Palaeoclimatology, Palaeoecology, vol. 206, no. 3-4, pp. 367-376, 2004.

[30] C. J. Krebs, "Niche measures and resource preferences," in Ecological Methodology, chapter 13, pp. 455-496, AddisonWesley Educational Publishers, 1999.

[31] StatSoft, "Statistica for Windows (data analysis software system)," version 7. 0, 2004, http://www.statsoft.com/.

[32] J. Codron, J. A. Lee-Thorp, M. Sponheimer, D. Codron, R. C. Grant, and D. J. de Ruiter, "Elephant (Loxodonta africana) diets in Kruger National Park, South Africa: spatial and landscape differences," Journal of Mammalogy, vol. 87, no. 1, pp. 27-34, 2006.

[33] P. van Wyk and N. Fairall, "The influence of the African elephant on the vegetation of the Kruger National Park," Koedoe, vol. 12, pp. 66-75, 1969.

[34] R. J. Scholes, W. J. Bond, and H. C. Eckhardt, "Vegetation dynamics in the Kruger ecosystem," in The Kruger Experience, J. T. Du Toit, K. H. Rogers, and H. C. Biggs, Eds., chapter 11, pp. 242-262, Island Press, Washington, DC, USA, 2003.

[35] T. G. O'Connor, P. S. Goodman, and B. Clegg, "A functional hypothesis of the threat of local extirpation of woody plant species by elephant in Africa," Biological Conservation, vol. 136, no. 3, pp. 329-345, 2007.

[36] C. V. Helm, E. T. F. Witkowski, L. Kruger, M. Hofmeyr, and N. Owen-Smith, "Mortality and utilisation of Sclerocarya birrea subsp. caffra between 2001 and 2008 in the Kruger National Park, South Africa," South African Journal of Botany, vol. 75, no. 3, pp. 475-484, 2009.

[37] G. Caughley and J. Goddard, "Abundance and distribution of elephants in the Luangwa Valley, Zambia," African Journal of Ecology, vol. 13, no. 1, pp. 39-48, 1975.

[38] S. K. Eltringham, "The numbers and distribution of elephants (Loxodonta africana) in the Rwenzori National Park and Chambura Game Reserve, Uganda," African Journal of Ecology, vol. 15, no. 1, pp. 19-39, 1977.

[39] T. A. Afolayan and S. S. Afayi, "The influence of seaso nality on the distribution of large mammals in the Yankari Game Reserve, 
Nigeria," African Journal of Ecology, vol. 18, no. 1, pp. 87-96, 1980.

[40] G. Merz, "Counting elephants (Loxodonta africana cyclotis) in tropical rain forests with particular reference to the Tai National Park, Ivory Coast," African Journal of Ecology, vol. 24, no. 2, pp. 133-136, 1986.

[41] P. J. Viljoen, "Habitat selection and preferred food plants of a desert-dwelling elephant population in the northern Namib Desert, South West Africa/Namibia," African Journal of Ecology, vol. 27, no. 3, pp. 227-240, 1989.

[42] M. N. Tchamba, "Number and migration patterns of savanna elephant (Loxodonta africana africana) in Northern Cameroon," Pachyderm, vol. 16, pp. 66-71, 1993.

[43] D. Babaasa, "Habitat selection by elephants in Bwindi Impenetrable National Park, South-Western Uganda," African Journal of Ecology, vol. 38, no. 2, pp. 116-122, 2000.

[44] G. P. Asner, S. R. Levick, T. Kennedy-Bowdoin et al., "Largescale impacts of herbivores on the structural diversity of african savannas," Proceedings of the National Academy of Sciences of the United States of America, vol. 106, no. 12, pp. 4947-4952, 2009.

[45] Y. Pretorius, J. D. Stigter, W. F. de Boer et al., "Diet selection of African elephant over time shows changing optimization currency," Oikos, vol. 121, no. 12, pp. 2110-2120, 2012. 

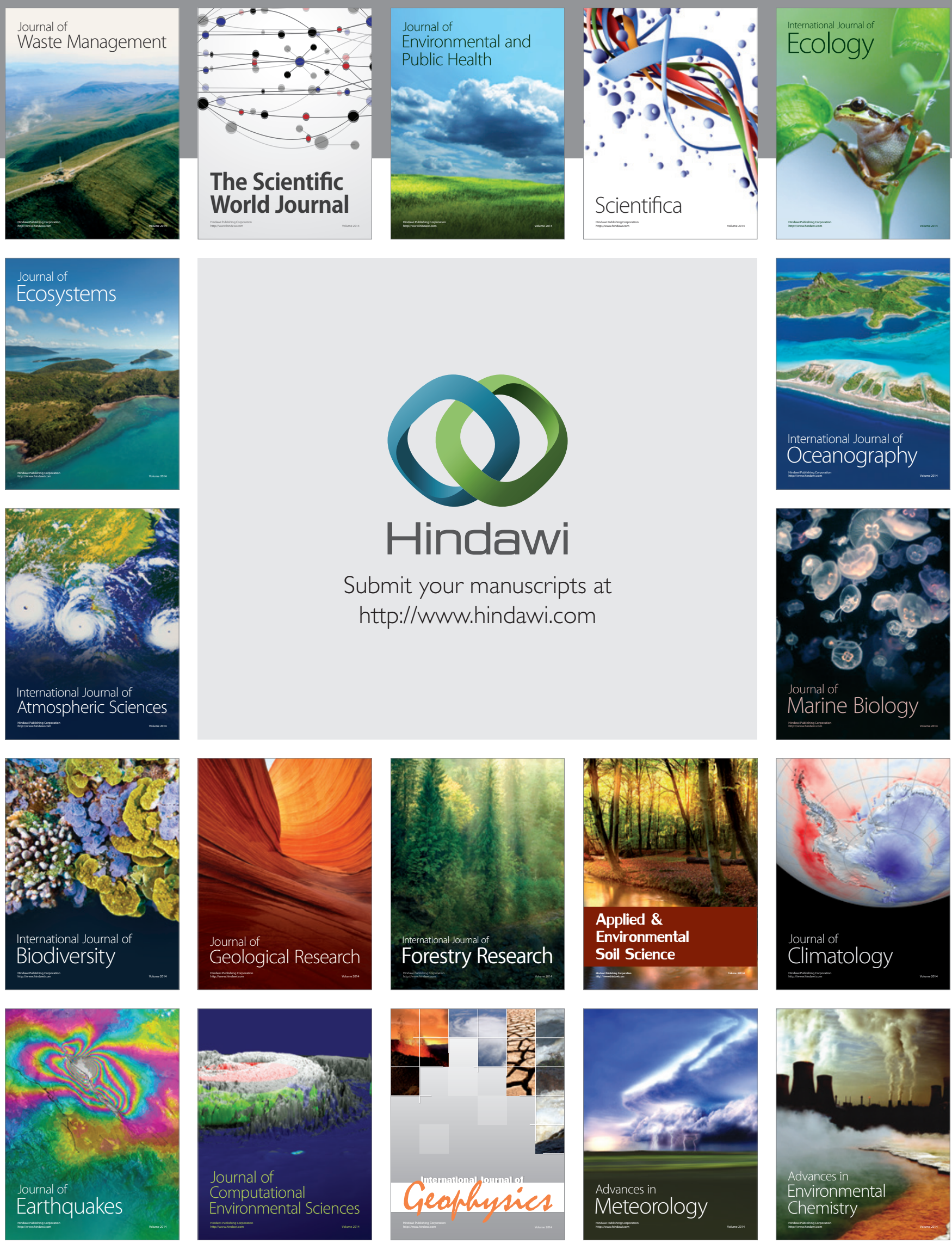